\title{
Connected Mobility Digital Ecosystem: A Case Study on Intelligent Transport Analytics
}

\author{
Edward Dou ${ }^{\mathrm{a}}$ \\ Peter Eklund $^{\mathrm{a}}$ \\ Tim Wray ${ }^{\mathrm{a}}$ \\ Chris Cook $^{\mathrm{a}}$ \\ Vu The Tran ${ }^{\mathrm{a}}$
}

\begin{abstract}
The UOWShuttle* is a transport app that provides users with real-time bus location and predictive information from Automatic Vehicle Location and Automatic Vehicle Counting systems. The system anonymously tracks interactions within the transport network. This paper is a briefing document on the analytics of the Connected Mobility Digital Ecosystem project, of which the UOWShuttle forms an important part. It describes its overall system architecture and gives some of the statistics of some of the data collected to date.
\end{abstract}

\section{Key words: Location-Based Services; Intelligent Transport Application; Digital Ecosystem.}

\section{Introduction: Location-based Services}

In this research we concentrate on a public transport passenger tracking application for a free shuttle bus network shown in Figure 1. This location data can be used to provide valueadded services to the users and manage system transport behavior including optimization of the transport resources and maximizing passenger comfort. While there are many issues in relation to the use of location-based applications, none are focused on as prolifically as the issue of privacy. Vicente, Freni, Bettini and Jensen ${ }^{1}$ and Rose ${ }^{2}$ claim that the most common way that information is released through these applications is by the users themselves. This is the approach adopted in our design with users opting in to having their location data recorded.

When considering location-based apps there are three main issues highlighted in the literature. These are the categorisation of applications, whether they are location tracking or location aware; the architecture of applications: including the location sensing technique and system architecture and thirdly matters of privacy. In our architecture, the clients are anonymised but each is allocated a unique identifier - related - but not identical - to the UUID of the users' device. This allows us to track when a device interacts with the transport network via the client app. We recorded the time, location and whether the passenger has joined a free bus and we can determine where they alight via an automatic passenger counting (APC) system that is installed on the doorways of all the shuttle buses in the fleet. We have no way of knowing the identity of the user but via this method we can determine if the same user interacts with the system multiple times.

\footnotetext{
a Faculty of Engineering and Information Sciences, University of Wollongong, Wollongong, NSW 2522, Australia

* The UOWShuttle app was written by Edward Dou and published by the Centre for Digital Ecosystems in November 2011. The UOWShuttleV2 app has undergone several improvements: version 2.3 of the UOWShuttleV2 app was published in September 2012. http://dx.doi.org/10.14453/isngi2013.proc.1
} 


\section{A. System Architecture}

In our system (Figure 2), the transport tracking system consists of four major components: on-board unit, which is installed on a vehicle (a bus); a vehicle presence server, responsible for receiving and processing data from on-board unit and saving it to the database server; a web server, which provides web services to the end users and matches data to a map; and finally a native iPhone application (the UOWShuttle client) for presentation to users.

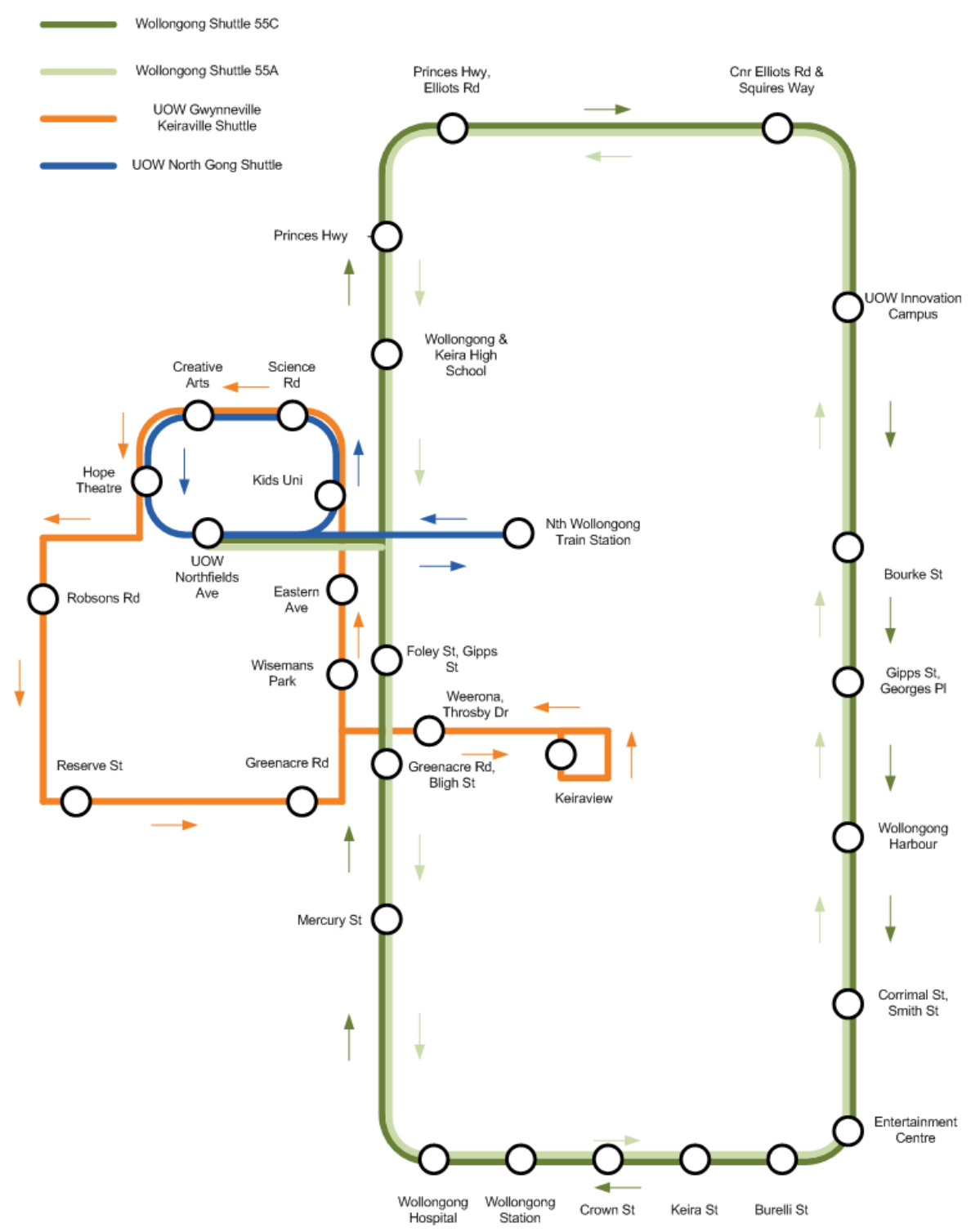

Figure 1. The Free Bus Network around the University of Wollongong forms the basis for the Connected Mobility Digital Ecosystem case study.

\section{B. On Board Unit}

The function of the in-vehicle device is to transmit shuttle bus's GPS coordinates and passenger usage information to a central server using a $3 \mathrm{G}$ cellular data link. The in-vehicle device also acts as a Wi-Fi hotspot. The Wi-Fi hotspot is a way of extending the digital footprint of the client by determining if their UUID is involved with sessioning via the app and/or connecting to the hotspot after boarding the bus. A central location server receives location updates from all in-vehicle devices and all buses in the fleet instrumented in this way. 


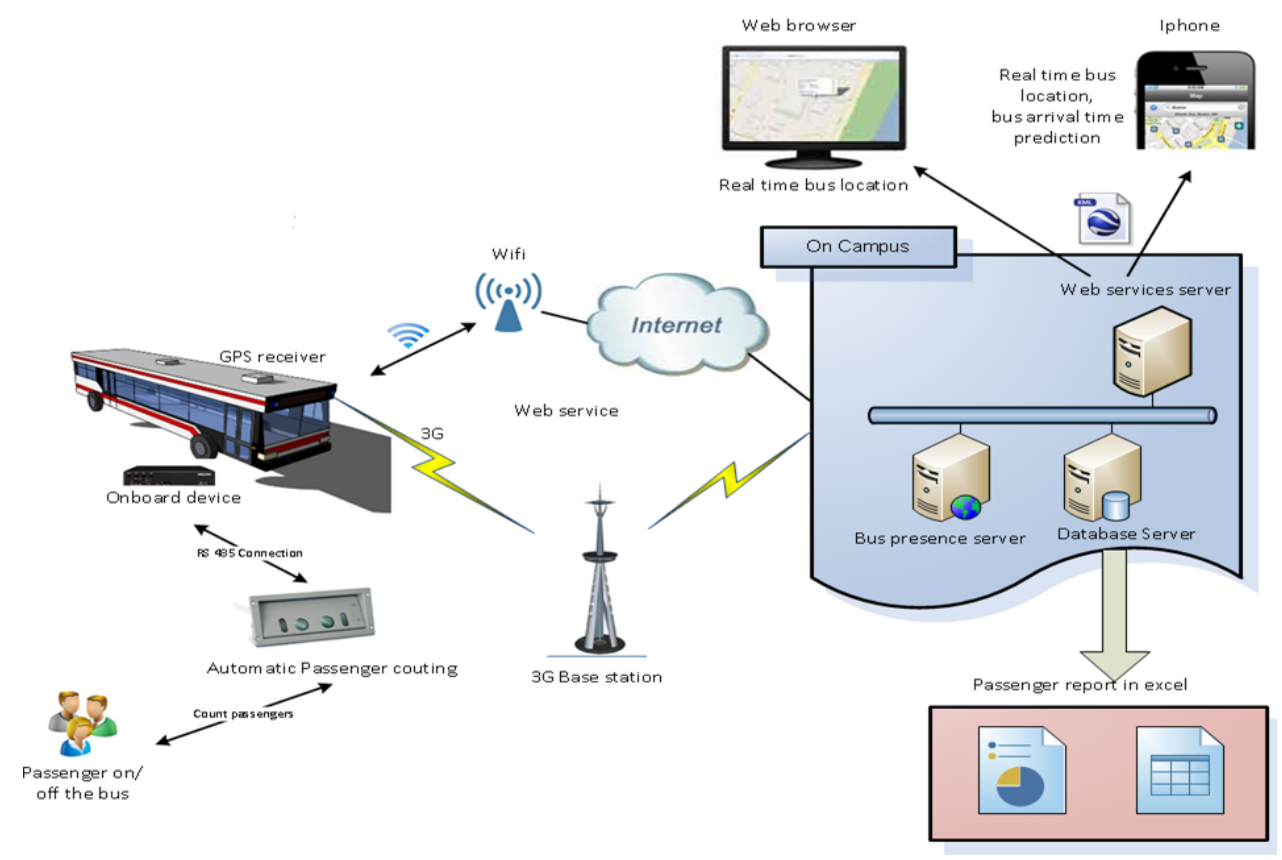

Figure 2. A Schematic of the Connected Mobility Transport Application Architecture.

The individual transactions are put through batch- and online processing. In batch mode, the vehicle GPS tracks are produce historic route timings, stop locations, and actual bus schedules. The bus passenger information produces daily passenger usage data. Online processing matches vehicles to routes, and performs arrival time prediction. An in-vehicle computer with built in GPS, 3G mobile and Wi-Fi is installed on the all buses in the fleet. A customized Linux OS with MySQL and Python is install on the system and is configured as a Wi-Fi hotspot. Internet access is provided to the passengers' device through the $3 \mathrm{G}$ data connection. An embedded application is also developed and installed on the in-vehicle unit. Its main purpose is to:

1. Capture the bus location.

2. Capture relevant bus passenger information.

3. Upload information to location server through the $3 \mathrm{G}$ connection.

4. Monitor data capturing and transmission processes, ensuring the processes are functioning at all times.

5. Monitor vehicle ignition for automatic system shutdown.

The following data capturing and transmission strategies are made:

1. After the system starts up and a 3G-connection is established, it establishes a secure SOAP/HTTPS link to the location server on campus.

2. Vehicle GPS coordinates, speed and heading are captured from a GPS unit and saved locally every second. This data is also uploaded to the vehicle presence server every 10 seconds through a secure link.

3. When passengers' Wi-Fi devices register and de-register with the in-vehicle hotspot, the GPS coordinates and time stamp of these events as well as the UUID derived unique identifier are captured. 


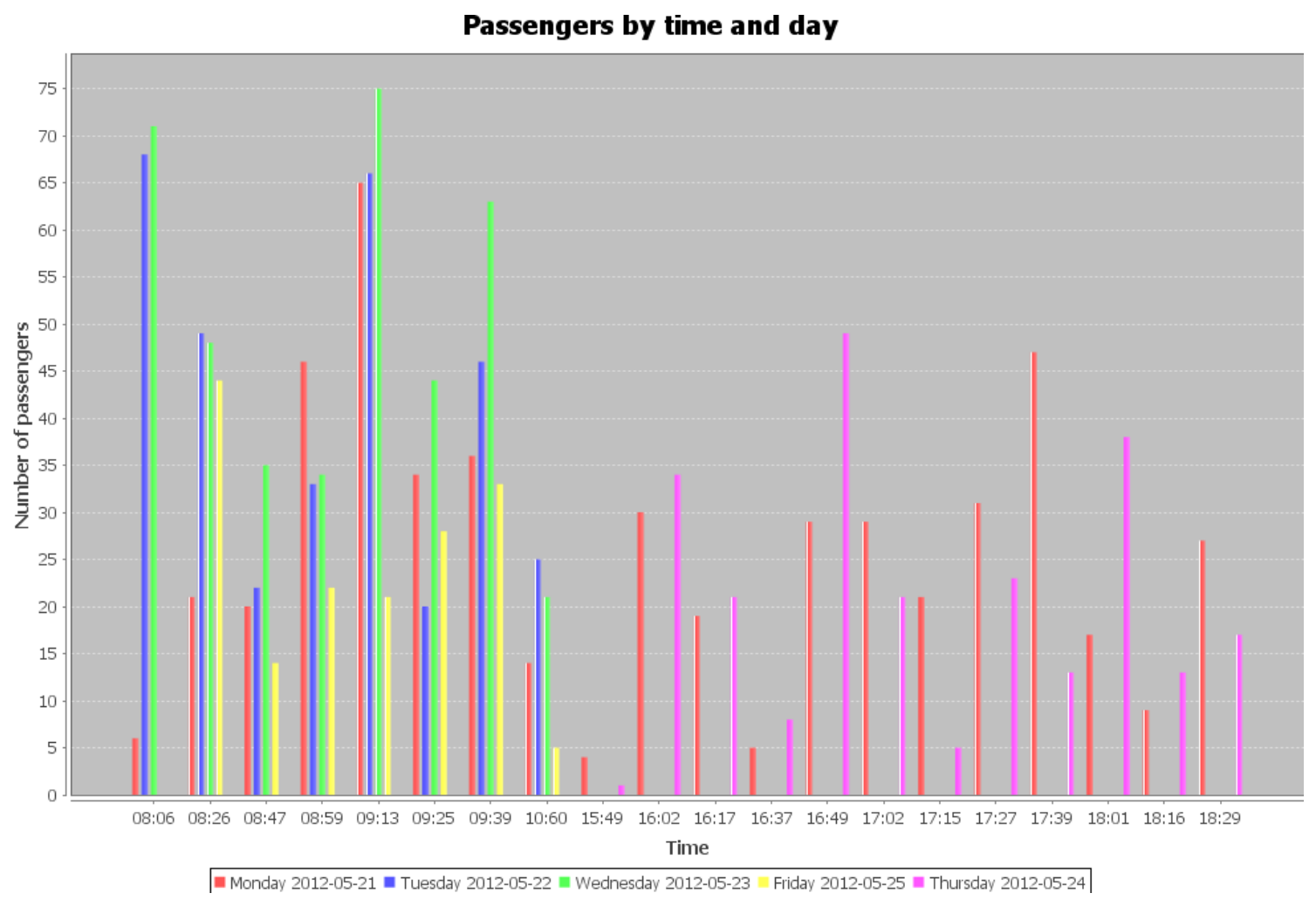

Figure 3. Passenger Usage Statistics for the Free Shuttle Bus Network at the University of Wollongong

The digital footprint of the passengers using the transport network is an important idea in the project. The challenge of capturing data about passenger movements is that the buses are free of charge. There are no tickets and therefore no knowledge of where passengers get on or off, as there would be in a ticketed transport system. Therefore, the question is how to automatically capture passenger movements via their interaction with technology on the buses. Capturing a digital footprint allows us to monitor trends in behavior over time and cross-reference the digital footprint with physical passenger behaviors as the technology adopts over time.

The passenger digital footprint is recorded in three ways. Firstly, by the number of passengers connecting to the on-board free Wi-Fi via a record of their UUID, secondly via the session data captured from the use of the transport app and thirdly via Automatic Passenger Counting (APC) devices installed on the bus doors.

In total, there are an average of 6,000 passenger events per month captured via IP and session tracking. The current number of app sessions recorded per month is between 7,00014,000 during the peak University teaching session and between 3,000-4,000 during vacation breaks. Not surprisingly, there is a correspondence between the use of the app and connections to the on-bus Wi-Fi. The average number of APC transactions - passenger on/off events - is 8,500 per month.

\section{Location Server Description}

A LAMP setup (Linux, Apache, MySQL, and PHP) is installed on a campus-based server. The server performs following functions:

1. Accepts secure SOAP connections from shuttle buses. 
2. Receives bus location data and bus passenger data through the secure SOAP link and stores this information into the MySQL database.

3. Processes bus travel timing information from the historical data and categorizes them into bus routes with 15 minutes segments.

4. Processes passenger UUID derived unique identifies and passenger counter information, maps these to the bus stop and scheduled trip information.

5. Predicates arrival information using current and historical data.

6. Provides current bus location and predicated arrival data.

7. Provides a Web interface to display the tracked vehicles.

8. Generates daily passenger usage reports for management.

9. Stores UOWShuttlev2 app usage information.

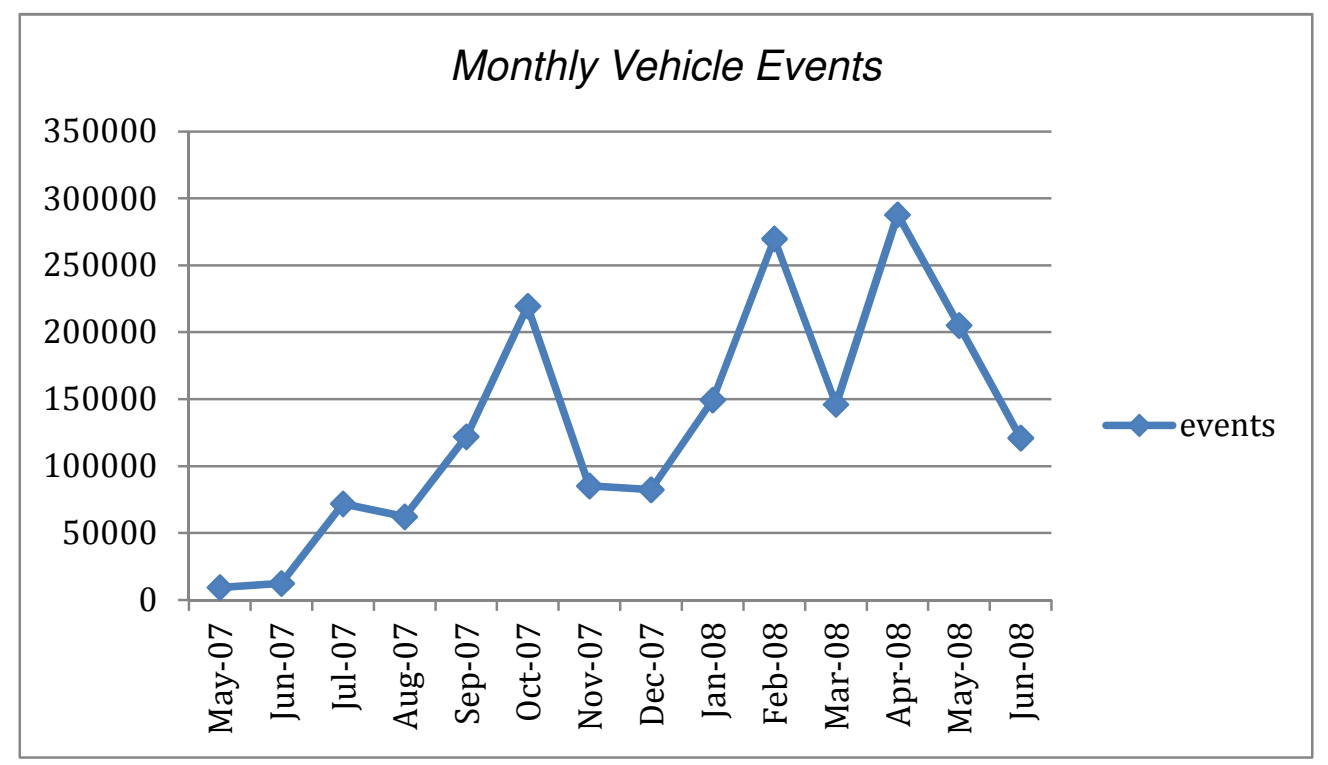

Figure 4. Monthly Vehicle Events

\section{The Data Warehouse}

The quantity of data collected by the project is already vast. The bus location data is updated to the server every 10 seconds for all the buses that are running at any one time. So far - as end of July 2013 - there were a total of 1,844,964 vehicle (bus) location events stored in a MySQL database on our servers. The average monthly number of vehicle events captured is 132,000 .

\section{E. The App: UOWShuttlev2}

UOWShuttlev2 is a native iOS iPhone app as is shown in Figure 5. It provides a location aware application for accessing real-time bus arrival information. It contains a 'table view' of stops for different routes with a counting down arrival time, a 'map view' showing the location of the user and the buses and a 'saved favourite trips' view. Usage statistics such as session time, user location and the UUID-based identifier of the iPhone are sent back to the server and stored. 


\section{Conclusion}

This paper describes the UOWShuttle app that provides users with real-time bus location and predicative arrival information from an Automatic Vehicle Location system. In return, the system tracks and warehouses user interactions with the app and within transport network. This paper gives a brief account of the data being collected for the project. The interactions between the physical and digital environments of the connected mobility system are complex enough to categorise the system as a digital ecosystem.
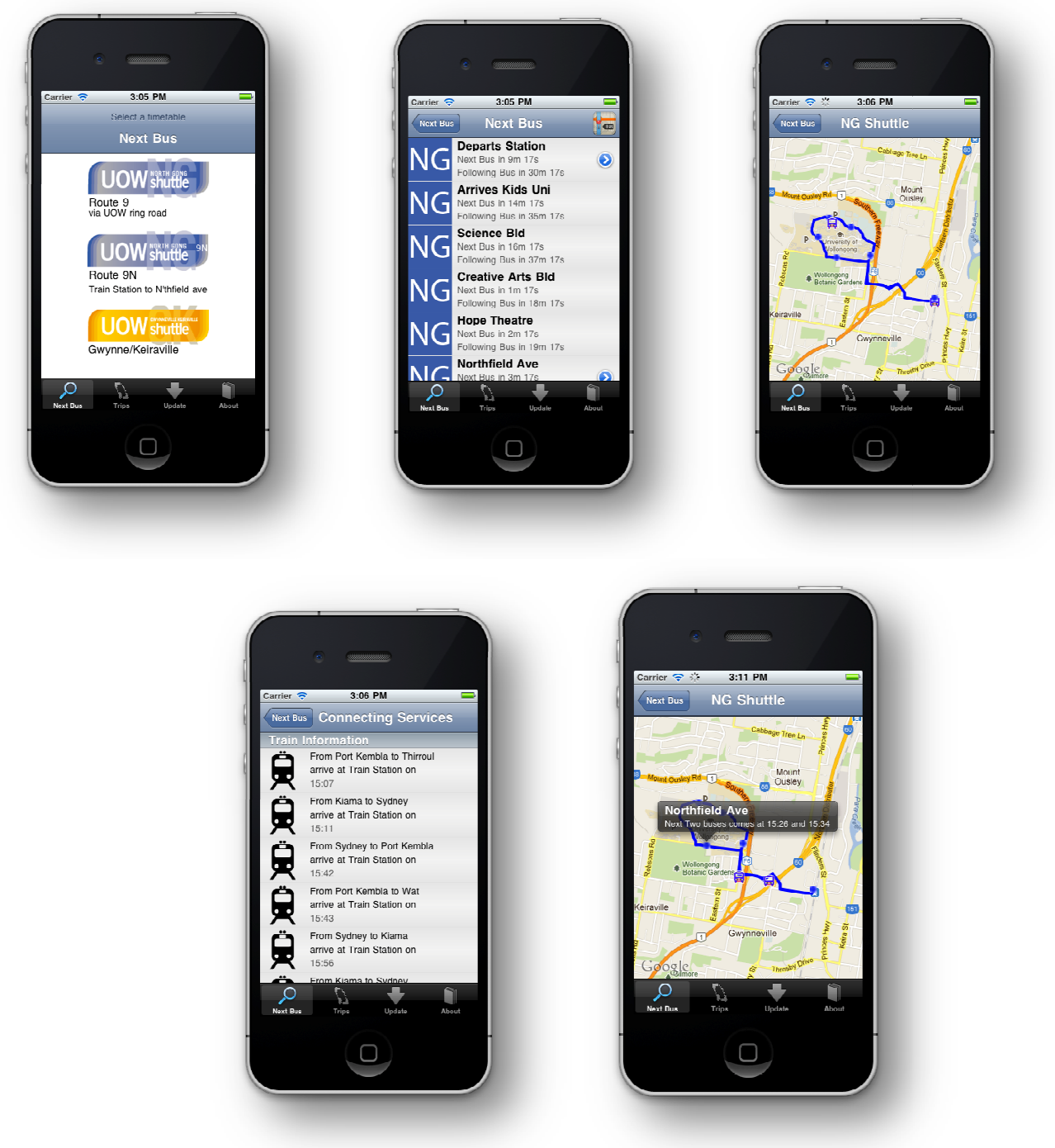

Figure 5. UOWShuttlev2 iPhone app: Nextbus, Map and Trip view screens.

\section{Acknowledgements}

This research has benefited from many individuals who have worked on the project, only a few of whom are acknowledged as co-authors. The project was supported by a cash grant from the CSIRO National ICT Centre and from the Australian Federal Government Award for Smart Infrastructure Research. 


\section{References}

${ }^{1}$ Vicente, C., Freni, D., Bettini, C., and Jensen, C., "Location-Related Privacy in Geo-Social Networks", IEEE Internet Computing, Vol. 15, No. 3, 2010, pp. 20-27. http://dx.doi.org/10.1109/MIC.2011.29

${ }^{2}$ Rose, C., "The Security Implications of Ubiquitous Social Media". EABR \& ETLC Conference Proceedings, Dublin, Ireland, 2010. 